\title{
Open-field behavior in domestic chicks tested individually or in pairs: Differential effects of painted lines delineating subdivisions of the floor
}

\author{
R. BRYAN JONES and NINA L. CARMICHAEL \\ Roslin Institute (Edinburgh), Roslin, Midlothian, Scotland
}

\begin{abstract}
The open field is a common test of fear, but the apparatus varies widely. Lines painted on the floor facilitate measurement of locomotion (areas entered), but, though some animals balk at lines, this variable has received little attention. In Experiment 1, 10-day-old female chicks were tested individually in an open field with an unmarked floor or one delineated into areas by 1- or 3-mm-wide black lines. In Experiment 2, chicks were tested in pairs in the presence or absence of 3-mm-wide lines. Strong intraindividual correlations demonstrated that both paces and areas entered are effective measures of locomotion. Chicks tested individually were unaffected by the presence or width of lines. Conversely, pair-tested chicks paced and pecked more when the floor was delineated. Given the latter findings, some standardization is recommended.
\end{abstract}

The open-field test has become established as a standard instrument in many laboratories, largely because of the ease and rapidity with which a number of basic behavior patterns can be measured. Its wide applicability has also contributed to its popularity; for example, open-field behavior is sensitive to environmental, experiential, pharmacological, and genetic manipulation. The test has been traditionally used to estimate fear (emotionality), although the interpretation of open-field behavior is a controversial issue. Indeed, researchers have evoked several behavioral constructs to explain their data; these have included fear, arousal, social motivation, predator evasion, exploration, and territorial marking (Archer, 1973; Gallup \& Suarez, 1980; Gray, 1979; Jones, 1987a, 1989; Walsh \& Cummins, 1976). However, these interpretations are not mutually incompatible, and it has been argued that fear may represent a common motivational state (Jones, 1989).

In most studies, the open field has consisted of a novel, barren, well-illuminated enclosure that is larger than the home cage and from which escape is prevented. However, this is a catholic definition. Many different types of apparatus have been used, and there has been considerable variation in shape, size, material, substrate, color, ambient noise, illumination, temperature, novelty value, and so on. Open-field behavior is sensitive to variations in all of these features (Jones, 1987a; Jones \& Faure, 1982; Walsh \& Cummins, 1976), and calls have been made for some standardization of the apparatus across laboratories (Walsh \& Cummins, 1976).

This study was commissioned and supported by the U.K. Ministry of Agriculture, Fisheries and Food. The Roslin Institute is supported by the Biotechnology and Biological Sciences Research Council. Correspondence should be addressed to R. B. Jones, Division of Environment and Welfare, Roslin Institute (Edinburgh), Roslin, Midlothian EH25 9PS, Scotland (e-mail: bryan.jones@bbsrc.ac.uk).
Some researchers (Archer, 1973; Walsh \& Cummins, 1976; Wimer \& Sterns, 1964) have also painted lines on the floor in order to delineate subdivisions or discrete areas of the open field and thereby to facilitate the measurement of one aspect of locomotor behavior in laboratory rats and mice (expressed as the number of areas entered). However, some domestic animals, such as cattle and pigs, are known to balk at and refuse to cross areas of high contrast on the floor, such as those created by shadows, plastic strips used as door thresholds, and drain grates (Grandin, 1994). Indeed, in the western United States, the highway department often paints lines across the road to prevent cattle from crossing (Grandin, personal communication, January 1995). Thus, the presence or absence of lines on the floor of the open field and differences in their nature may represent other potentially confounding sources of variation between laboratories. However, to the best of our knowledge, the potential effects of such lines on open-field behavior had been neglected until Greenberg (1994) demonstrated that the width of such lines influenced ambulation in spiny mice.

Although the bulk of open-field research has focused on laboratory rodents, the domestic chick is also receiving growing attention in this respect. Indeed, because fear can seriously damage the welfare, management, and performance of poultry (Jones, 1996), its assessment and reduction are important. Furthermore, it has been suggested that the open-field responses of young chicks may represent potential selection criteria for future commercial breeding programs (Faure, 1980). Here, however, there has also been considerable diversity in the types of open field used in different laboratories (Jones, 1989), and the floors either have remained unmarked or have been subdivided by white or black lines into squares, rectangles, circles, or various other shapes (Archer, 1976; Faure, 1981; Gallup, Ledbetter, \& Maser, 1976; Jones \& 
Waddington, 1992; Sanberg, Faulks, Anson, \& Mark, 1982). The nature of the substrate used in the open field is already known to be influential, with chicks showing greater ambulation when it consisted of wood shavings or wire mesh rather than paper (Jones \& Black, 1979). Lines painted on the floor might also represent a particularly influential variable because not only are domestic chicks capable of fine visual discrimination but they generally pay close attention to the ground and readily peck at conspicuous or discrepant stimuli (Dawkins, 1968; Rogers, 1995). However, there is no information concerning the potential effects of painted lines on their openfield behavior.

The present study addressed this issue in two separate experiments. Chicks are most commonly tested in isolation in the open field. Therefore, in Experiment 1, we recorded the open-field responses of individually tested 10-day-old female chicks as a function of the presence or absence of black lines painted on a gray floor and of their width $(1$ or $3 \mathrm{~mm})$. However, chicks are also frequently tested in pairs, trios, or even larger groups (Jones, 1989). The presence of social companions in the open field has been reported to reduce fear, to modify vocalization and locomotory behavior, and to increase pecking at food and nonfood items by domestic chicks (Hogan \& Abel; 1971; Jones, 1983, 1989; Jones \& Merry, 1988; Tolman, 1965; Wilson, 1968). In this context, it could then be argued that the presence of lines might be more likely to elicit exploratory pecking and to thereby indirectly affect locomotion when birds are tested socially. Therefore, in Experiment 2, we compared the open-field responses of pairs of chicks when they were tested in the presence or absence of 3-mm-wide black lines on the otherwise gray floor.

Because sex differences have been reported in the responses of domestic chicks exposed to an open field or to sudden isolation (Jones, 1987b; Jones \& Faure, 1982; Jones \& Williams, 1992; Vallortigara \& Zanforlin, 1988), an arbitrary choice was made, and only female chicks were used. The chicks were always tested when they were 10 days old. This age was chosen because, as overall bias to right hemisphere control develops, fear levels are thought to become relatively stable from Day 9 or 10 onwards (Andrew \& Brennan, 1984). Our choice of a circular open field with a gray floor painted with black lines reflected the type of apparatus that we have typically used in our laboratory. Furthermore, beginning with Hall (1934), open fields have most commonly been circular.

\section{GENERAL METHOD}

\section{Subjects and Husbandry}

Female ISA Brown chicks (a medium-hybrid line originally derived from a Rhode Island Red $\times$ Rhode Island White cross) were used in both experiments. The chicks were obtained in two batches (48 in Experiment 1, and 56 in Experiment 2) from a commercial supplier at 1 day of age. Upon receipt, they were allocated at random to one of three groups of 16 or 20 chicks. The groups were then housed in separate compartments within the upper and middle decks of a three-deck tier brooder. The bottom deck was not used because of the low levels of illumination and extraneous stimulation to which its residents would have been exposed. Food (chick starter mash) was provided ad lib in a trough at the front of the compartment, and water was freely available from nipple drinkers situated along one side of each compartment. The photoperiod ran from 0500 to $1900 \mathrm{~h}$, and the ambient temperature was maintained at approximately $29^{\circ} \mathrm{C}$. The chicks remained undisturbed apart from routine maintenance activities until they were tested at 10 days of age.

\section{Open-Field Apparatus and Test Room}

The open field was situated in a room different from that in which the chicks were housed, and it consisted of a 75-cm-diameter circular arena. The $45-\mathrm{cm}$-high metal wall was painted matt black, whereas the wooden floor was colored pale gray. Three different types of floor were used in Experiment 1. The first was unmarked (U). The second was marked by a circular black line (indelible ink) that divided the floor into an inner area and an outer area and by three black lines drawn across the diameter and at $60^{\circ}$ to each other. These lines subdivided the floor into 12 areas of approximately equivalent size; all lines were $1 \mathrm{~mm}$ wide (OF1). The third type of floor was similarly subdivided by black lines, but these were $3 \mathrm{~mm}$ wide (OF3). In Experiment 2, only the U and OF3 floors were used.

The ambient temperature in the test room during both experiments was similar to that under which the chicks were housed. Ventilator fans ran continuously in both the housing and the test rooms, and they provided similar levels of what might be construed as white noise.

\section{EXPERIMENT 1}

\section{Method}

Each chick was tested individually and once only at 10 days of age. It was picked at random from one of the three brooder compartments, carried approximately $5 \mathrm{~m}$ by hand, and placed in the center of the open field. A video camera was suspended $2 \mathrm{~m}$ directly above the open field, and the chick's behavior was recorded for subsequent analysis using a closed-circuit television system. The three test conditions (U, OF 1 , and OF3) were imposed in random order, and 16 chicks were tested in each. A diagrammatic representation of the open-field subdivisions was also prepared on an acetate sheet; this was placed on the television screen during analysis of responses in the $U$ test situation. The following measures were recorded over the 5-min observation period: the latencies to vocalize and to ambulate, and the numbers of vocalizations, paces, areas entered, jumps, pecks at the environment, and defecations. The chick was deemed to have moved from one area to another when the major part of its body had crossed the line. The chick was marked with indelible ink and then returned to its home cage after testing; the mark ensured that it was not inadvertently retested. If defecation had occurred, the floor of the open field was cleaned with paper tissues before the next trial.

Comparisons of the behavioral measures were made across each of the three test conditions using the Kruskal-Wallis analysis of variance (adjusted for ties). The Mann-Whitney $U$ test (two-tailed) was used for between-test comparisons if these were deemed necessary. The degree of intraindividual association between the two scores of locomotor behavior (areas entered and paces) was determined using pooled data and the Spearman rank correlation coefficient.

\section{Results}

Apart from jumping, the chicks' open-field responses were not affected either by the presence or absence or by 
Table 1

Open-Field Responses of Individually Tested Female Domestic Chicks When the Floor Either was Unmarked $(U)$ or was Delineated Into Sectors by 1-mm-wide (OF1) or 3-mm-wide (OF3) Black Lines

\begin{tabular}{|c|c|c|c|c|c|c|c|}
\hline \multirow[b]{2}{*}{ Measure } & \multicolumn{2}{|c|}{$\mathrm{U}$} & \multicolumn{2}{|c|}{ OF 1} & \multicolumn{2}{|c|}{ OF3 } & \multirow[b]{2}{*}{$p$} \\
\hline & $M$ & $S E$ & $M$ & $S E$ & $M$ & $S E$ & \\
\hline \multicolumn{8}{|l|}{ Latency (in seconds) } \\
\hline to vocalize & 25.4 & 18.5 & 26.8 & 18.5 & 40.8 & 25.3 & .44 \\
\hline Latency (in seconds) & & & & & & & \\
\hline to ambulate & 48.9 & 19.6 & 104.7 & 29.4 & 67.8 & 26.3 & .18 \\
\hline \multicolumn{8}{|l|}{ Number of : } \\
\hline Vocalizations & 388.4 & 35.4 & 279.5 & 40.1 & 290.3 & 41.6 & .10 \\
\hline Paces & 28.3 & 4.7 & 40.1 & 12.8 & 33.9 & 10.5 & .85 \\
\hline Areas entered & 5.9 & 0.8 & 5.0 & 1.1 & 5.2 & 1.0 & .60 \\
\hline Jumps & 1.5 & 0.8 & 0.6 & 0.4 & 0.4 & 0.2 & .04 \\
\hline Pecks & 2.7 & 1.1 & 1.5 & 1.1 & 0.2 & 0.2 & .09 \\
\hline Defecation & 1.3 & 0.1 & 1.0 & 0.2 & 1.1 & 0.2 & .49 \\
\hline
\end{tabular}

Note- $p$ values derived using the Kruskal-Wallis analysis of variance.

the width of the lines painted on the floor (Table 1). Jumping was observed more often in the $U$ test situation than in either the OF1 $(U=78, p<.039)$ or the OF3 $(U=$ $76, p<.032)$ tests. This finding is considered likely to reflect chance, particularly since only 19 of the 48 chicks tested actually jumped ( 11 in U, 4 in OF1, and 4 in OF3). Of these, most jumped only once; however, the $U$ group contained 1 outlier that jumped 12 times.

A high intraindividual correlation $\left(r_{s}=.946 ; N=48\right.$; $t=6.782 ; p<.001)$ was found between the numbers of paces and of areas entered.

\section{EXPERIMENT 2}

\section{Method}

Pairs of chicks were captured from one of the three home compartments and immediately carried to the test room. One of the chicks was marked on its head with indelible ink in order to facilitate identification. They were then placed simultaneously in the center of the open field. Two types of floor were used on this occasion. These were either the unmarked gray floor or one subdivided into 12 sectors with 3-mm-wide black lines. Fourteen pairs of chicks were tested in each of these situations. The chicks' responses were recorded on videotape over a 5-min period, and the variables described above were again measured for each of the 2 chicks. The previously unmarked chick was spotted with ink before both were returned to the brooder.

For each variable measured, the scores recorded for each member of a pair were summed and then halved to give a single value for each pair. The resultant pair means were then used as data points. The Mann-Whitney $U$ test was used to compare chicks' responses in the two test situations. The intraindividual association between the numbers of paces and of areas entered was calculated using pooled data and the Spearman rank correlation coefficient.

\section{Results}

Paired chicks paced and pecked at the environment significantly more when they were tested in an open field with a delineated floor rather than an unmarked floor (Table 2). A numerical tendency to enter more areas was also apparent in the presence of lines. Jumps are not reported in Table 2 because this behavior was observed in only 1 of the 56 chicks tested, and this chick jumped only once. In those trials where pecking was observed in the lined open field, the chicks showed similar $(p=.773)$ numbers of pecks at the black lines per se $(4.5 \pm 0.92$, mean and standard error) and at the general environment $(4.0 \pm 0.77)$.

Scores of walking and of areas entered were positively and highly correlated within individual birds (pooled data; $r_{s}=.900 ; N=28 ; t=5.096 ; p<.001$ ).

\section{DISCUSSION}

Although subdividing the floor of an open field with painted lines in order to aid the measurement of locomotor activity is a common practice, its potential role as an influential variable had been virtually neglected. However, the apparent interplay found in the present study between this variable and the social situation at test sounds a cautionary note, at least in the domestic chick.

On the one hand, apart from jumping, the open-field responses of socially housed, individually tested chicks

Table 2

Responses of Socially Reared Female Domestic Chicks When They Were Tested in Pairs in an Open Field with an Unmarked Floor or One That was Delineated Into Sectors by 3-mm-wide Black Lines

\begin{tabular}{|c|c|c|c|c|c|}
\hline \multirow[b]{2}{*}{ Measure } & \multicolumn{2}{|c|}{ Unmarked } & \multicolumn{2}{|c|}{ Lined } & \multirow[b]{2}{*}{$p$} \\
\hline & $M$ & $S E$ & $M$ & $S E$ & \\
\hline \multicolumn{6}{|l|}{ Latency (in seconds) } \\
\hline to vocalize & 89.1 & 23.4 & 59.2 & 22.1 & .46 \\
\hline \multicolumn{6}{|l|}{ Latency (in seconds) } \\
\hline to ambulate & 134.1 & 34.0 & 107.6 & 18.5 & .25 \\
\hline \multicolumn{6}{|l|}{ Number of: } \\
\hline Vocalizations & 178.4 & 30.1 & 119.1 & 24.5 & .66 \\
\hline Paces & 11.1 & 4.4 & 26.1 & 5.6 & .03 \\
\hline Areas entered & 2.5 & 0.4 & 3.6 & 0.5 & .09 \\
\hline Pecks & 0.50 & 0.23 & 3.50 & 0.08 & .005 \\
\hline Defecation & 0.32 & 0.09 & 0.25 & 0.09 & .52 \\
\hline
\end{tabular}

Note $-p$ values derived from analysis by the Mann-Whitney $U$ test (two-tailed). 
were unaffected by the presence or width of black lines painted on the gray floor. Jumping was observed more often when chicks were tested on an unmarked $(U)$ floor; however, in view of the low number of birds that showed this behavior and the presence of an obvious outlier in the $U$ treatment group, this finding probably reflects a chance effect. Collectively, these findings suggest that rigid standardization of the presence or width of lines delineating subdivisions may not be required when measuring the open-field behavior of individually tested domestic chicks. Whether this conclusion generalizes across different ages, strains, and species, across different shapes, sizes, and colors of open field, or even across different numbers, colors, and shapes of lines remains to be determined. However, it is interesting to note that Wimer and Sterns (1964) found no differences in locomotor activity when mice were tested either in a visually simple open field that had been divided into squares with black lines or in one that had been made visually complex by the addition of outlined geometric forms within the squares.

On the other hand, pacing and pecking at the environment were observed significantly more often when pairs of chicks were tested in an open field with a delineated floor rather than an unmarked floor. Open-field ambulation often reflects attempts to escape from the enclosure or to reinstate social contact with the home group (Gallup \& Suarez, 1980; Jones, 1987a). However, given the virtual absence of jumping and the numerical tendency toward reduced vocalization, the high levels of environmental pecking shown in the presence of lines are more consistent with an interpretation based on heightened exploration rather than social reinstatement or escape. Furthermore, pecking was directed not only at the lines but also to other areas of the floor and the wall.

Strong intraindividual correlations were found in both experiments between the numbers of paces and of areas entered. These correlations suggest that both these scores are effective measures of locomotor behavior. These findings are also reminiscent of the significant association reported (Faure \& Jones, 1981) when the open-field behavior of domestic chicks was recorded automatically (number of light beams broken) and by direct observation (number of paces).

In conclusion, although individually tested chicks were unaffected, the presence of lines on the floor influenced open-field behavior when chicks were tested in pairs. The latter finding suggests that standardization of this variable across laboratories could eliminate at least one potential confound and thereby aid the interpretation of open-field data. Standardization could be easily and effectively achieved in one of two ways. First, if closedcircuit television is available, the floor of the open field could be remotely subdivided by placing an acetate sheet marked with a grid pattern over the monitor screen. Second, in view of the positive association found here between the scores of pacing and of areas entered, simply counting the number of paces should yield effective measures of ambulation.

\section{REFERENCES}

ANDREw, R. J., \& BRENNAN, A. (1984). Sex differences in lateralization in the domestic chick. Neuropsychologia, 33, 503-509.

ARCHER, J. (1973). Tests for emotionality in rats and mice: A review. Animal Behaviour, 21, 205-235.

ARCHER, J. (1976). Testosterone and fear behavior in male chicks. Phvsiology \& Behavior, 17, 561-564.

DAWKINS, R. (1968). The ontogeny of a pecking preference in domestic chicks. Zeitschrift für Tierpsychologie, 25, 170-186.

FAURE, J. M. (1980). To adapt the environment to the bird or the bird to the environment? In R. Moss (Ed.), The laving hen and its environment (pp. 19-42). Brussels: EEC.

FAURE, J. M. (1981). Bidirectional selection for open-field activity in young chicks. Behavior Genetics, 11, 135-144.

Faure, J. M., \& Jones, R. B. (1981). A comparison of automatic recording and direct observation of open-field behaviour in domestic chicks. IRCS Medical Science, 9, 112-113.

Gallup, G. G., JR., Ledbetter, D. H., \& Maser, J. D. (1976). Strain differences among chickens in tonic immobility: Evidence for an emotionality component. Journal of Comparative \& Physiological Psychology, 90, 1075-1081.

Gallup, G. G., JR., \& Suarez, S. D. (1980). An ethological analysis of open-field behaviour in chickens. Animal Behaviour, 28, 368-378

Grandin, T. (1994, October). Solving livestock handling problems. Veterinary Medicine, pp. 989-998.

GraY, J. A. (1979). Emotionality in male and female rodents: A reply to Archer. British Journal of Psychology, 70, 425-440.

GREENBERG, G. (1994, July). The open field test in comparative psychology: A critical analysis. Paper presented at the VIIth Meeting of the International Society for Comparative Psychology, São Paulo, Brazil.

HaLL, C. S. (1934). Emotional behavior in the rat: 1. Defecation and urination as measures of individual differences in emotionality. Journal of Comparative Psychology, 18, 385-403.

Hogan, J. A., \& ABEL, E. L. (1971). Effects of social factors on response to unfamiliar environments in Gallus gallus spadiceus. Animal Behaviour, 19, 687-694.

JONES, R. B. (1983). Fear responses in domestic chicks as a function of the social environment. Behavioural Processes, 8. 309-325.

JONES, R. B. (1987a). The assessment of fear in the domestic fowl. In R. Zayan \& I. J. H. Duncan (Eds.), Cognitive aspects of social behaviour in the domestic fowl (pp. 40-81). Amsterdam: Elsevier.

JONES, R. B. (1987b). Social and environmental aspects of fear in the domestic fowl. In R. Zayan \& I. J. H. Duncan (Eds.), Cognitive aspects of social behaviour in the domestic fowl (pp. 82-149). Amsterdam: Elsevier.

JONES, R. B. (1989). Avian open-field research and related effects of environmental novelty: An annotated bibliography, 1960-1988. Psychological Record, 39, 397-420.

JONES, R. B. (1996). Fear and adaptability in poultry: Insights, implications and imperatives. World's Poultry Science Journal, 52, 131-174

JONES, R. B., \& BLACK, A. J. (1979). The behaviour of domestic chicks in their home cages and in an open field: Substrate effects. IRCS Medical Science, 7, 169.

JONES, R. B., \& FAURE, J. M. (1982). Open-field behaviour of male and female domestic chicks as a function of housing conditions, test situations and novelty. Biology of Behaviour, 7, 17-25.

JONES, R. B., \& MERRY, B. J. (1988). Individual or paired exposure of domestic chicks to an open field: Some behavioural and adrenocortical consequences. Behavioural Processes, 16, 75-86.

Jones, R. B., \& WADDINGTON, D. (1992). Modification of fear in domestic chicks, Gallus gallus domesticus, via regular handling and early environmental enrichment. Animal Behaviour, 43, 1021-1033.

JONES, R, B., \& WiLliams, J. B. (1992). Responses of pair-housed male and female domestic chicks to the removal of a companion. Applied Animal Behaviour Science, 32, 375-380. 
ROGERS, L. J. (1995). The development of brain and behaviour in the chicken. Wallingford, U.K.: CAB International.

SAnBERG, P. R., FAulKs, I. J., ANSON, J. M., \& MARK, R. F. (1982). Operant, open-field, and tonic immobility behaviors in chickens with forebrain injections of cycloheximide or glutamate. Behavioural Brain Research, 4, 19-32.

Tolman, C. W. (1965). Emotional behaviour and social facilitation of feeding in domestic chicks. Animal Behaviour, 13, 493-502.

VALLORTIGARA, G., \& ZANFORLIN, M. (1988). Open-field behavior of young chicks (Gallus gallus): Antipredatory responses, social reinstatement motivation, and gender effects. Animal Learning \& Behavior, 16, 359-362.
Walsh, R. N., \& Cummins, R. A. (1976). The open-field test: A critical review. Psychological Bulletin, 83, 482-504.

WILSON, G. F. (1968). Early experience and facilitation of feeding in domestic chicks. Journal of Comparative \& Physiological Psychology, 66, 800-802.

Wimer, R., \& Sterns, H. (1964). Controlled visual input and exploratory activity in C57BL/6J mice. Perceptual \& Motor Skills, 18, 299-307.

(Manuscript received October 31, 1995; revision accepted for publication July 29, 1996.) 